\title{
Evaluation of percutaneous dilatational tracheostomy under laryngosuspension
}

\author{
Mathieu Moulin ${ }^{1} \cdot$ Marie-Pierre Aboussouan $^{2} \cdot$ Paul F. Castellanos $^{3} \cdot$ Ihab Atallah $^{1,4} \mathbb{D}$
}

Received: 11 May 2020 / Accepted: 16 June 2020 / Published online: 23 June 2020

(c) Springer-Verlag GmbH Germany, part of Springer Nature 2020

\begin{abstract}
Purpose To describe the technique and outcomes of percutaneous tracheostomy under laryngosuspension.

Methods A consecutive series of patients who underwent percutaneous tracheostomy under laryngosuspension were reviewed for outcomes. The procedure is performed under general anesthesia and laryngosuspension. An 8.5 oral endotracheal tube is inserted and the cuff is inflated in the supraglottis, allowing access to the whole trachea and subglottis. The tube is taped to the laryngoscope. A rigid endoscope is inserted in the endotracheal tube through a swivel connector connected to the tube and the ventilation circuit. Percutaneous tracheostomy kit is used to perform dilatational tracheostomy at the desired level of the trachea under direct visual endoscopic control.

Results Forty-eight patients underwent percutaneous tracheostomy under laryngosuspension. Thirty-two cases were performed in an emergency after securing the airway with endotracheal intubation. In all other cases, tracheostomy was performed as a part of an elective procedure affecting the airway or in patients requiring prolonged ventilation. More than half of patients were considered high risk by virtue of one or more of the following: morbid obesity, prior neck surgery, prior neck radiotherapy, progressive head and neck cancer, or laryngotracheal stenosis. Complications occurred in seven cases. Conclusion Percutaneous tracheostomy under laryngosuspension has the advantage of optimal control of patient ventilation and hemostasis throughout the procedure. High-quality endoscopic vision and easy access to the airway under laryngosuspension allow tracheostomy to be performed with maximum safety.
\end{abstract}

Keywords Laryngosuspension $\cdot$ Tracheostomy $\cdot$ Percutaneous tracheostomy $\cdot$ Endoscopy $\cdot$ Airway

\section{Introduction}

Paul F. Castellanos and Ihab Atallah senior authors for this manuscript.

Ihab Atallah

iatallah@chu-grenoble.fr

Mathieu Moulin

mmoulin5@chu-grenoble.fr

Marie-Pierre Aboussouan

mpaboussouan@chu-grenoble.fr

Paul F. Castellanos

voxdoxster@gmail.com
Tracheostomy is an ancient surgical procedure, described over 4000 years ago. Chevalier Jackson in the early twentieth century described a safer surgical technique with the addition of anesthesia and modern surgery [1]. This technique, performed through a cervical incision providing direct

2 Department of Anesthesiology and Intensive Care Medicine, Grenoble Alpes University Hospital, BP 217, 38043 Grenoble Cedex 09, France

3 Otolaryngology-Head and Neck Surgery, Northern Light Health Medical Center, Bangor, ME 04401-3073, USA

4 School of Medicine, Grenoble Alpes University, Domaine de la Merci, BP 170 La Tronche, 38042 Grenoble Cedex 09, France

1 Otolaryngology-Head and Neck Surgery Department, Grenoble Alpes University Hospital, BP 217, 38043 Grenoble Cedex 09, France 
approach to the trachea, has continued to improve and has become a technique of reference.

Shelden made the first attempts of percutaneous tracheostomy in 1955 [2]. Percutaneous tracheostomy with a dilatation system was proposed by Ciaglia in 1985 [3]. It then became a safe technique and was adopted as an alternative to conventional surgical tracheostomy. It is characterized by the use of a dilatation kit and endoscopic control during the procedure [4-6]. This procedure is mainly performed in intensive care units, in patients requiring prolonged ventilation [7]. Percutaneous tracheostomy has several advantages over conventional surgical tracheostomy: reduced operative time, reduced risk of intra- and postoperative bleeding, reduced risk of infection of the surgical site, lower cost, and reduced time of spontaneous tracheostomy stoma closure after decannulation. The overall complication rate of both techniques seems to be identical [8-10]. Several contraindications to percutaneous tracheostomy have been reported: pediatric population, unsafe airway, emergency, presence of a median cervical lesion, non-palpable cricoid cartilage, and coagulopathy [5]. Airway emergencies are characterized by urgency and unpredictability that need a clear process to be followed so that the airway can managed rapidly [11]. The following factors further increase the difficulty of emergency airway management: (I) lack of information on detailed medical history without enough time to get complete thorough physical examination before needing to act; (II) lack of preparation: the decision to establish an artificial airway is often made suddenly and preparation time is usually short, which means a comprehensive preparation cannot be relied upon at every intubation; (III) lack of compliance: emergency patients may have difficulty in cooperating [11].

Percutaneous dilatational tracheostomy under laryngosuspension is a blending of two techniques $[12,13]$. In this technique, ventilation and hemostasis are secured during the entire procedure with permanent and easy access to the airway in case of complication [12, 13]. Here, we present an evaluation of this technique that allows tracheostomy to be performed with maximal safety in the setting of emergency or elective procedure.

\section{Material and methods}

A cohort of all patients undergoing percutaneous dilatational tracheostomy under laryngosuspension from 2016 to 2019 was retrospectively reviewed at a tertiary referral otolaryngology center. Informed consent for data collection in medical records was obtained. All procedures performed in the study were in accordance with the ethical standards of the institution and with the 1964 Helsinki Declaration and its later amendments. In addition, our study got the approval of the national committee of data privacy (Commission nationale de l'Informatique et des Libertés pour l'Utilisation des Données Patients, approval number: 2205066v0).

Recorded data consisted of age, sex, body mass index (BMI), tracheostomy indication, perioperative complications such as pre or postoperative desaturation $\left(\mathrm{SpO}_{2}<90 \%\right)$, bleeding, and accidental decannulation occurring within five days following the procedure. In addition, history of tracheostomy, neck surgery, laryngotracheal stenosis, and coagulopathy were collected. The size of the tracheostomy tube and the operative time were also reported.

Intubation criteria collected were Mallampati score, maximum mouth opening, thyromental distance in centimeters, cervical spine mobility, and Cormack-Lehane grade. In addition, short cricosternal distance in extended position, corresponding to the absence of a gap between the inferior border of the cricoid cartilage and the sternum, was reported. Intubation modalities included direct laryngoscopy, videolaryngoscopy using the GlideScope ${ }^{\circledR}$ (Verathon, Bothell, WA, USA) or the C-MAC ${ }^{\circledR}$ videolaryngoscope (Karl Storz, Tuttlingen, Germany), rigid bronchoscopy, and flexible transnasal bronchoscopy.

\section{Operative technique}

Percutaneous dilatational tracheostomy technique under laryngosuspension was described in details in one of our previous works [12].

In brief, after securing the airway with adequate size endotracheal tube in the setting of emergency or elective procedure, a Bouchayer laryngoscope and arm suspension apparatus (Integra ${ }^{\circledR}$ MicroFrance ${ }^{\circledR}$, Saint Aubin le Monial, France), a $5.5 \mathrm{~mm} 0^{\circ}$ Storz rigid endoscope (Karl Storz Endoscopy Ltd., Tuttlingen, Germany), and an 8.5 oral endotracheal tube were used for laryngotracheal examination and airway control.

An ULTRAperc ${ }^{\circledR}$, Portex ${ }^{\circledR}$ tracheostomy introducer (Smiths-Medical, Kent, UK) is used in all cases. Patient ventilation is maintained throughout the entire procedure with only very brief intervals of apnea during endotracheal tube exchange and airway evaluation. The laryngoscope is placed just into the laryngeal inlet within the aperture of the false vocal folds. An 8.5 oral endotracheal tube is inserted and the cuff is inflated and impacted in the supraglottis to avoid any air leak, allowing access to the whole trachea and subglottis as well as adequate ventilation of the patient during percutaneous tracheostomy performance. The tube is taped to the laryngoscope to avoid any displacement. The rigid endoscope is inserted in the endotracheal tube through a swivel connector connected to the tube and the ventilation circuit. Percutaneous tracheostomy kit is then used to perform dilatational tracheostomy at the desired level of the trachea under direct visual endoscopic control (Fig. 1). At the end of the procedure, a Blue Line Ultra ${ }^{\circledR}$ size 7 or 8 


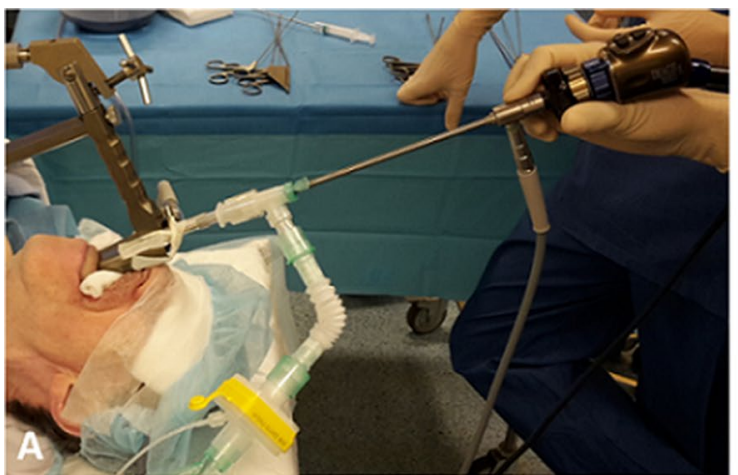

Fig. 1 Percutaneous dilatational tracheostomy under laryngosuspension. a Patient is placed in rigid suspension and intubated through the laryngoscope with an 8.5 endotracheal tube. The patient is venti-

Table 1 Indications for percutaneous dilatational tracheostomy under laryngosuspension

\begin{tabular}{lrr}
\hline Indications & $n$ & $\%$ \\
\hline Elective head and neck cancer surgery $^{c}$ & 3 & 6 \\
Scheduled airway surgery & 7 & 15 \\
Malignant upper airway obstruction $^{\mathrm{a}}$ & 17 & 35 \\
Non-malignant airway obstruction $^{\mathrm{a}}$ & 15 & 31 \\
Prolonged ventilation & 5 & 10 \\
Swallowing disorders with aspiration & 1 & 2 \\
\hline
\end{tabular}

${ }^{\mathrm{a}}$ Tracheostomy was performed in an emergency setting

(Smiths-Medical, Kent, UK) tracheostomy is inserted and stitched to the skin to reduce the risk of accidental decannulation. The laryngoscope and the endotracheal tube are removed.

\section{Results}

Percutaneous dilatational tracheostomy under laryngosuspension was performed in 48 cases (36 males and 12 females). The mean age was 63 years. The mean BMI was $22.7 \pm 4.8$. No patient had a BMI $>35$.

Indications for tracheostomy are represented in Table 1. Sixty-seven percent of cases were performed in an emergency setting of malignant $(n=17)$ and non-malignant $(n=15)$ upper airway obstruction after securing the airway with endotracheal intubation. Non-malignant airway obstruction etiologies included laryngeal stenosis secondary to radiotherapy or surgery $(n=5)$, cricoid chondronecrosis secondary to radiotherapy $(n=4)$, bilateral vocal fold paralysis in adduction due to central or peripheral etiologies, head and neck cellulitis associated with dyspnea $(n=1)$, laryngeal abscess in a patient with immunosuppression $(n=1)$, and autoimmune inflammatory laryngitis $(n=1)$.

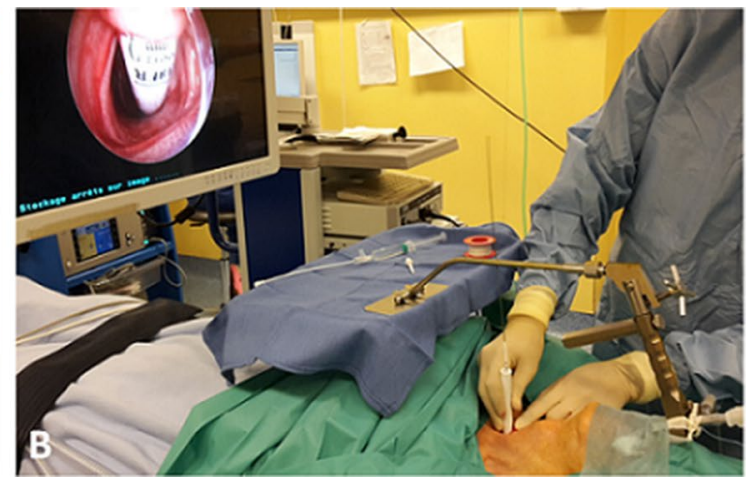

lated throughout the procedure via the side port. b A rigid telescope inserted into the endotracheal tube offers high quality endoscopic vision throughout the entire procedure

Intubation criteria included a median Mallampati score of 2 and a mean maximum mouth opening of $2.8 \pm 0.8$ fingers. The thyromental distance was greater than $6.5 \mathrm{~cm}$ in 30 cases (63\%), less than $6.5 \mathrm{~cm}$ in 2 cases $(4 \%)$, and was missing in the medical records in 16 cases. Cervical spine mobility was decreased in 11 patients $(23 \%)$ and was normal in 21 patients (4\%). Data concerning cervical spine mobility were missing in the medical records of sixteen patients. The median Cormack-Lehane score was 2 and data were missing in thirty-nine cases.

Intubation modalities included direct laryngoscopy in 33 cases (69\%), videolaryngoscopy in 9 cases (19\%), and flexible transnasal bronchoscopy in 6 cases (13\%). There was no use of rigid bronchoscopy for intubation in any case.

Short cricosternal distance was reported in 5 cases (10\%). Eleven patients (23\%) had a history of tracheostomy and 14 patients (29\%) had a history of neck surgery. Twenty cases (46\%) had a history of head and neck radiotherapy. No history of coagulopathy was found.

Size 8 tracheostomy tube was inserted in 29 patients (60\%) and size 7 in 19 patients (40\%).

Major complications occurred in three cases (Table 2). Significant postoperative bleeding occurred in one case and needed surgical revision for hemostasis. Postoperative accidental decannulation occurred in two cases within 5 days after the procedure: one case required general anesthesia to reinsert the tracheostomy tube at the operating room (OR) and one patient died secondary to cardiopulmonary arrest. Oral intubation was possible but the patient refused resuscitation in accordance to his advance directives. Minor complications occurred in four cases. One case presented peroperative desaturation $\left(\mathrm{SpO}_{2}<90 \%\right)$ due to abundant bronchial secretions that were suctioned during the procedure. Insignificant peroperative bleeding occurred in two cases and was stopped after inserting the tracheostomy tube. Post-operative desaturation $\left(\mathrm{SpO}_{2}<90 \%\right)$ was reported in one case due to 
Table 2 Complications of percutaneous dilatational tracheostomy under laryngosuspension

\begin{tabular}{|c|c|c|}
\hline Complications & $n$ & $\%$ \\
\hline \multicolumn{3}{|l|}{ Minor complications } \\
\hline Peroperative desaturation $\left(\mathrm{SpO}_{2}<90 \%\right)$ & 2 & 4 \\
\hline Postoperative desaturation $\left(\mathrm{SpO}_{2}<90 \%\right)$ & 1 & 2 \\
\hline Minor peroperative bleeding & 1 & 2 \\
\hline \multicolumn{3}{|l|}{ Major complications } \\
\hline Postoperative bleeding $^{\mathrm{a}}$ & 1 & 2 \\
\hline Accidental decannulation & 2 & 4 \\
\hline Total & 7 & 14 \\
\hline
\end{tabular}

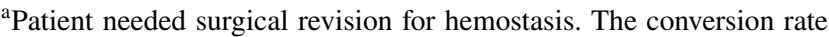
to open tracheostomy was $2 \%$

abundant bronchial secretions and resolved on suction. The mean operative time was $14 \pm 3 \mathrm{~min}$.

\section{Discussion}

Percutaneous dilatational tracheostomy under laryngosuspension can be performed in the context of emergency or elective surgery. Two thirds of our procedures were performed in an emergency setting of acute dyspnea, which was previously considered as a contraindication to dilatational percutaneous tracheostomy. In our series, first, the airway was accessed with the appropriate intubation modality then percutaneous dilatational tracheostomy under laryngosuspension was performed. This needs appropriate experience with all intubation modalities in order to be prepared to manage any difficult airway to avoid performance of awake open tracheostomy. The latter is considered as a stressful condition for the patient, the surgeon and the anesthesiologist in case of any delay in securing the airway due to distorted neck anatomy or difficult surgical approach to the trachea. In most of emergency cases of our series, the airway was first intubated with a small endotracheal tube and then laryngosuspension was performed allowing permanent access to the airway during the whole procedure and safe endotracheal tube exchange with a larger one (ID: 8.5) permitting adequate ventilation of the patient while inserting a rigid telescope in the endotracheal tube. In traditional percutaneous dilatational tracheostomy with flexible endoscopic control, smaller endotracheal tubes are used and this may result in ventilatory compromise secondary to partial obstruction of the small tubes by the endoscope. In addition, laryngosuspension allows easy examination of the airway and the performance of biopsies in case of malignant airway obstruction. Finally, laryngosuspension and taping the endotracheal tube to the laryngoscope allows retraction of the distal end of the tube to the laryngeal inlet and inflation of its cuff in the supraglottis, without any fear to lose the airway, thus permitting visualization of the majority of the airway starting from the subglottis. No damage or injury to the true vocal folds occurred in our series as the balloon of the endotracheal tube was inflated within the supraglottis and only the distal tip of the tube was inserted at the level of the glottis.

In our series, percutaneous dilatational tracheostomy under laryngosuspension was performed in the OR while most of the series of percutaneous tracheostomy under laryngosuspension described in the literature are performed in the intensive care unit (ICU) in patients requiring prolonged ventilation $[10,13]$. The advantage of performing the procedure in the OR is the presence of a technical platform that allows dealing with any complication. In addition, it allows easy access to flexible and rigid instrumentation for intubation with maximal security of patients with airway emergencies. The disadvantage remains a higher cost than a procedure performed in the ICU [8]. In contrast, we have to remind that in the actual context of COVID-19 pandemic, percutaneous dilatational tracheostomy under laryngosuspension could be an alternative to open tracheostomy in patients with prolonged ventilation secondary to acute respiratory distress syndrome, not candidate for traditional percutaneous tracheostomy. In this setting, percutaneous dilatational tracheostomy under laryngosuspension could be performed in ICU to avoid transfer of infected patients to the OR to diminish the risk of COVID-19 spread during transportation.

In our series, airway exposure scores were satisfactory, although this could not be confirmed in cases with missing data, but the difficulty remained in finding the airway and to insert the endotracheal tube due to the high proportion of malignant airway obstruction and distorted airway anatomy secondary to radiotherapy and previous airway surgeries. Our series is different from those described in the literature where percutaneous tracheostomy is mainly performed in patient already intubated requiring prolonged ventilation in the ICU $[13,14]$.

Most obese patients have very short, thick necks. Obesity makes for challenging airway access regardless of the technique used. Palpation of anatomic landmarks often reveals a short cricostenal distance. In addition, neck extension might not help or could be limited. Open surgical tracheostomy in these patients are notoriously difficult with the trachea oriented obliquely and posteriorly within the thorax. Percutaneous dilatational tracheostomy under laryngosuspension is far easier and safer than open tracheostomy or conventional percutaneous tracheostomy in these patients. In addition, it might require the use of an extended length tracheostomy cannula which is not usually supplied with the set of percutaneous tracheostomy [15]. The surgeon should always be prepared to introduce an endotracheal tube, either through the laryngoscope from 
above or directly into the wound itself, in the event that passage of the tracheostomy tube is not initially successful [13]. This is facilitated by the laryngosuspension that provides stable and easy access to the airway. Although we have been ready for this possibility, thus far it has not been necessary. In our series, $27 \%$ of patients were overweight with a maximum BMI of 33.3. No patient was morbidly obese (BMI > 35). We did not encounter any particular difficulty in patients with overweight. Percutaneous dilatational tracheostomy under laryngosuspension appears to be a good alternative in overweight patients. This is confirmed in American series with a mean BMI of 28.1 $[13,15,16]$.

In our series, no patients had coagulation disorders. However, percutaneous tracheostomy could be performed in patients with bleeding disorders defined as INR $>1.5$, or platelet count $<50,000 / \mathrm{mm}^{3}$, or receiving curative anticoagulation treatment with heparin $[16,17]$. Laryngosuspension allows endoscopic control of hemostasis in case of bleeding using large-bore suction rigid instrumentation, tamponade, and monopolar or bipolar electrocautery.

Three major complications (6\%) occurred in our series. This complication rate is comparable to that of other teams using similar technique and appears to be lower than conventional percutaneous tracheostomy techniques despite the great heterogeneity of the average rates of complications estimated between 9.2 and $37.4 \%$ in published series $[10,13,15,18,19]$. In our series, complications occurred in patients with no intubation difficulties and with various tracheostomy indications. In our experience, we did not find any risk factor predictive for complications. In general, the main complication in percutaneous tracheostomy is inadvertent decannulation which enables the tip of the cannula to fall out entirely or to sit within a false passage anterior to the tracheal lumen [8]. For this reason, we recommend to stitch the tracheostomy tube to the skin and to perform the first cannula change at least on post-operative day 5. In our series, no complications were observed regarding posterior tracheal wall injury during the procedure. This could be explained by the fact that the rigid telescope provides improved direct optical visualization. Finally, complications such as pneumothorax or major subcutaneous emphysema did not occur in our series.

Despite broadening of our indications for percutaneous dilatational tracheostomy under laryngosuspension, there are still few contraindications such as severe trismus, unstable cervical spine fractures, and patients contraindicated to general anesthesia. Severe trismus prevents introduction of the laryngoscope for laryngosuspension. Unstable cervical spine fractures is a contraindication for laryngosuspension due to the risk associated with neck extension. Conventional percutaneous dilatational tracheostomy is preferable in this context [20].
In our series, a single expert airway surgeon performed all the procedures. The senior author recommends performing five to ten procedures accompanied by a mentor, before that otolaryngologists interested in this technique, get autonomy to practice it [14]. Once mastered, this technique presents a short operative time comparing to open surgical tracheostomy.

We did not perform neck ultrasound check-up before the procedure. This seems to be a point to work on for the additional safety that ultrasound could provide regarding anterior cervical vascular structures localization [21, 22]. Ultrasound guided localization of the trachea seems to be less convincing in our technique. Indeed great extension of the neck provided by laryngosuspension, improves ability to palpate anatomical airway landmarks [12]. In addition, rigid endoscope helps to see palpation on the anterior tracheal wall to visualize and centralize the planned puncture site before cannula insertion.

We propose this technique as an alternative for open tracheostomy in ICU patients not candidate for conventional percutaneous tracheostomy. These patients include cases with unsafe airway, midline neck mass, inability to palpate the cricoid cartilage and uncorrectable coagulopathy [5]. The laryngosuspension system allows direct access to the upper airway for efficient ventilation with a high level of safety and visualization quality during the entire surgery with the possibility to perform complementary hemostasis and aspiration throughout the procedure.

Any conventional percutaneous dilatational tracheostomy should continue to be performed in the ICU after careful evaluation of its feasibility to avoid transportation of critically ill patients to the OR and unnecessary costs $[5,14]$.

\section{Conclusion}

Percutaneous dilatational tracheostomy under laryngosuspension is an improvement of preexisting conventional percutaneous tracheostomy. It is an alternative to open surgical tracheostomy in many indications. It can be performed in the setting of airway emergency or as a part of an elective surgery affecting the airway. It has the advantage of optimal control of the patient ventilation and hemostasis throughout the procedure. High quality endoscopic vision and easy access to the airway under laryngosuspension allow tracheostomy to be performed with maximum safety.

Author contributions All authors contributed significantly to the work, have seen and approved the manuscript.

Funding No source of funding. 
Availability of data and material The data that support the findings of this study are available on request from the corresponding author.

\section{Compliance with ethical standards}

Conflict of interest The authors declare that they have no conflict of interest.

Ethics approval All procedures performed in studies involving human participants were in accordance with the ethical standards of the institutional and with the 1964 Helsinki declaration and its later amendments or comparable ethical standards. Our study got the approval of the national committee of data privacy (Commission nationale de l'informatique et des Libertés pour l'Utilisation des Données Patients, approval number: 2205066v0).

Consent to participate Informed consent was obtained from all patients for participation in this study.

Consent for publication Informed consent was obtained from all patients for publication of the study.

Code availability N/A.

\section{References}

1. Jackson C (1909) Tracheotomy. Laryngoscope 19(4):285-290

2. Shelden CH, Pudenz RH, Freshwater DB, Crue BL (1955) A new method for tracheotomy. J Neurosurg 12(4):428-431. https://doi. org/10.3171/jns.1955.12.4.0428

3. Ciaglia P, Firsching R, Syniec C (1985) Elective percutaneous dilatational tracheostomy. A new simple bedside procedure; preliminary report. Chest 87(6):715-719. https://doi.org/10.1378/ chest.87.6.715

4. Byhahn C, Wilke HJ, Halbig S, Lischke V, Westphal K (2000) Percutaneous tracheostomy: ciaglia blue rhino versus the basic ciaglia technique of percutaneous dilational tracheostomy. Anesth Analg 91(4):882-886. https://doi.org/10.1097/00000539-20001 $0000-00021$

5. Kost KM (2005) Endoscopic percutaneous dilatational tracheotomy: a prospective evaluation of 500 consecutive cases. Laryngoscope 115(10 Pt 2):1-30. https://doi.org/10.1097/01.MLG.00001 63744.89688.E8

6. Shen G, Yin H, Cao Y, Zhang M, Wu J, Jiang X, Yu T, Lu W (2019) Percutaneous dilatational tracheostomy versus fibre optic bronchoscopy-guided percutaneous dilatational tracheostomy in critically ill patients: a randomised controlled trial. Ir J Med Sci 188(2):675-681. https://doi.org/10.1007/s11845-018-1881-3

7. Vargas M, Sutherasan Y, Antonelli M, Brunetti I, Corcione A, Laffey JG, Putensen C, Servillo G, Pelosi P (2015) Tracheostomy procedures in the intensive care unit: an international survey. Crit Care 19:291. https://doi.org/10.1186/s13054-015-1013-7

8. Higgins KM, Punthakee X (2007) Meta-analysis comparison of open versus percutaneous tracheostomy. Laryngoscope 117(3):447-454. https://doi.org/10.1097/01.mlg.0000251585 $.31778 . c 9$
9. Freeman BD, Isabella K, Lin N, Buchman TG (2000) A metaanalysis of prospective trials comparing percutaneous and surgical tracheostomy in critically ill patients. Chest 118(5):1412-1418. https://doi.org/10.1378/chest.118.5.1412

10. Oliver ER, Gist A, Gillespie MB (2007) Percutaneous versus surgical tracheotomy: an updated meta-analysis. Laryngoscope 117(9):1570-1575. https://doi.org/10.1097/MLG.0b013e3180 93edae

11. Sun F, Wang Y, Ma S, Zhu H, Yu X, Xu J, Chinese Collaboration Group for Emergency Airway M (2017) Clinical consensus of emergency airway management. J Thorac Dis 9(11):4599-4606. https://doi.org/10.21037/jtd.2017.10.79

12. Moulin M, Castellanos P, Atallah I (2020) Percutaneous tracheostomy under suspension laryngoscopy. Eur Ann Otorhinolaryngol Head Neck Dis (in press)

13. White HN, Sharp DB, Castellanos PF (2010) Suspension laryngoscopy-assisted percutaneous dilatational tracheostomy in highrisk patients. Laryngoscope 120(12):2423-2429. https://doi. org/10.1002/lary.21019

14. Hsia DW, Ghori UK, Musani AI (2013) Percutaneous dilational tracheostomy. Clin Chest Med 34(3):515-526. https://doi. org/10.1016/j.ccm.2013.04.002

15. Sharp DB, Castellanos PF (2009) Clinical outcomes of bedside percutaneous dilatational tracheostomy with suspension laryngoscopy for airway control. Ann Otol Rhinol Laryngol 118(2):91-98. https://doi.org/10.1177/000348940911800203

16. Blankenship DR, Kulbersh BD, Gourin CG, Blanchard AR, Terris DJ (2005) High-risk tracheostomy: exploring the limits of the percutaneous tracheostomy. Laryngoscope 115(6):987-989. https ://doi.org/10.1097/01.MLG.0000163107.80668.12

17. Kluge S, Meyer A, Kühnelt P, Baumann HJ, Kreymann G (2004) Percutaneous tracheostomy is safe in patients with severe thrombocytopenia. Chest 126(2):547-551

18. Nowak A, Kern P, Koscielny S, Usichenko TI, Hahnenkamp K, Jungehulsing M, Tittel M, Oeken J, Klemm E (2017) Feasibility and safety of dilatational tracheotomy using the rigid endoscope: a multicenter study. BMC Anesthesiol 17(1):7. https://doi. org/10.1186/s12871-017-0301-y

19. Nowak A, Klemm E (2011) Percutaneous dilatational tracheotomy using the tracheotomy endoscope. Laryngoscope 121(7):14901494. https://doi.org/10.1002/lary.21849

20. Ben Nun A, Orlovsky M, Best LA (2006) Percutaneous tracheostomy in patients with cervical spine fractures-feasible and safe. Interact Cardiovasc Thorac Surg 5(4):427-429. https://doi. org/10.1510/icvts.2005.125690

21. Rudas M, Seppelt I, Herkes R, Hislop R, Rajbhandari D, Weisbrodt L (2014) Traditional landmark versus ultrasound guided tracheal puncture during percutaneous dilatational tracheostomy in adult intensive care patients: a randomised controlled trial. Crit Care 18(5):514. https://doi.org/10.1186/s13054-014-0514-0

22. Yavuz A, Yilmaz M, Goya C, Alimoglu E, Kabaalioglu A (2014) Advantages of US in percutaneous dilatational tracheostomy: randomized controlled trial and review of the literature. Radiology 273(3):927-936. https://doi.org/10.1148/radiol.14140088

Publisher's Note Springer Nature remains neutral with regard to jurisdictional claims in published maps and institutional affiliations. 